Western University Scholarship@Western

Centre for Decision Sciences and Econometrics

Technical Reports

Economics Working Papers Archive

1985

\title{
On Formulating Wald Tests of Nonlinear Restrictions
}

\author{
Allan W. Gregory \\ Michael R. Veall
}

Follow this and additional works at: https://ir.lib.uwo.ca/economicscdse_tr Part of the Economics Commons

Citation of this paper:

Gregory, Allan W., Michael R. Veall. "On Formulating Wald Tests of Nonlinear Restrictions." Centre for Decision Sciences and Econometrics Technical Reports, 2. London, ON: Department of Economics, University of Western Ontario (1985). 


\title{
THE CENTRE FOR DECISION SCIENCES AND ECONOMETRICS
}

On Formulating Wald Tests of Nonlinear Restrictions

Allan W. Gregory and Michael R. Veall

TECHNICAL REPORT NO. 2

JUNE 1985

\author{
Centre For Decision Sciences And Econometrics \\ Social Science Centre \\ The University of Western Ontario \\ London, Ontario N6A 5C2
}


ON FORMULATING WALD TESTS OF NONLINEAR RESTRICTIONS*

\author{
Allan W. Gregory and Michael R. Veall \\ Department of Economics \\ University of Western Ontario \\ London \\ Canada
}

Revised September 1984

\begin{abstract}
This note discusses a problem which has received little attention in the econometrics literature, namely that Wald tests of nonlinear restrictions that are algebraically equivalent under the null hypothesis give different results in small samples. The magnitude of potential discrepancies is illustrated by a simple Monte Carlo experiment.
\end{abstract}

* The authors would like to thank G. Fisher, E. Leamer, D. Levine, A. Melino, C. R. Rao, M. Samps on, R. Tibshirani, A. U1lah, members of workshops at the University of Western Ontario and UCLA and two anonymous referees for helpful comments and Mark McCann and Carolan Miller for research assistance. Earlier versions were presented at the meetings of the Canadian Economics Association in Guelph, Ontario, May 1984, and at the European meetings of the Econometric Society in Madrid, Spain, September 1984. Both authors acknowledge financial support from the Social Sciences and Humanities Research Council of Canada. 
1. Introduction

As opposed to the Likelihood Ratio (IR) and Lagrange Multiplier (LM) or score tests, the Wald test is most convenient when the unrestricted model is easier to estimate than the restricted one, because the restricted model is linear but the restriction is nonlinear. Unfortunately, for this case, the Wald test has a drawback which does not appear to have been studied in the econometrics literature. The problem is that in small samples, changing the form of a nonlinear restriction to a form which is algebraically equivalent under the null hypothesis will change the numerical value of the Wald test statistic, although it will not change the LR or LM tests. This is because the Wald test is derived from a Taylor series expansion (see, for example, Silvey, 1975) and different ways of writing equivalent nonlinear expressions lead to nontrivial differences in the corresponding Taylor series. Therefore, although all the different Wald tests have the same asymptotic distribution, in actual practice there could be conflicts. While this has been noted directly (e.g., Burguete, Gallant and Souza, p. 185, 1982) and indirectly (e.g., Cox and Hinkley, p. 302, 1974), the purpose of this note is to show, on the basis of Monte Carlo evidence, that differences in the functional form of the nonlinear restrictions are Iikely to be important in the small sample sizes often encountered in economics.

\section{An Illustrative Example}

Consider the following classical linear regression equation model in $\mathrm{N}$ observations:

$$
y_{t}=\beta_{0}+\beta_{1} x_{1 t}+\beta_{2} x_{2 t}+\epsilon_{t},
$$


where $y_{t}$ is the dependent variable, $x_{1 t}$ and $x_{2 t}$ are exogenous variables, $\beta_{0}$ is a constant, $\beta_{1}$ and $\beta_{2}$ are slope coefficients not equal to zero and $\varepsilon_{t}$ is iid $\left(0, \sigma^{2}\right)$. Under these assumptions, ordinary least squares (OLS) yields the best linear unbiased estimator.

Suppose one wishes to test the hypothesis that one parameter is equal to the reciprocal of the other:

$$
H_{0}^{A}: \quad g^{A}\left(\beta_{1}, \beta_{2}\right)=\beta_{1}-1 / \beta_{2}=0
$$

An alternative form to equation (2) which is algebraically equivalent under the null hypothesis is:

$$
\mathrm{H}_{0}^{\mathrm{B}}: \quad \mathrm{g}^{\mathrm{B}}\left(\beta_{1}, \beta_{2}\right)=\beta_{1} \beta_{2}-1=0
$$

The general formula of the Wald statistic is

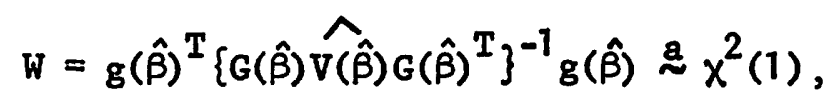

where $g$ is the restriction expressed either as in equation (2) or equation (3), $G(\beta)=\partial g(\beta) / \partial \beta^{T}$ is evaluated at $\hat{\beta}$, the ols estimator of $\beta, \widehat{V(\hat{\beta})}$ is the usual estimated variance-covariance matrix of $\hat{B}$. The test has the asymptotic distribution of a chi-square (with one degree of freedom because there is only one restriction). Using (4), the two alternative Wald test statistics can be calculated as:

$$
W^{A}=\left(\hat{\beta}_{1} \hat{\beta}_{2}-1\right)^{2} /\left(\hat{\beta}_{2}^{2} v_{11}+2 v_{12}+v_{22} / \hat{\beta}_{2}^{2}\right)
$$

and $\quad W^{B}=\left(\hat{\beta}_{1} \hat{\beta}_{2}-1\right)^{2} /\left(\hat{\beta}_{2}^{2} v_{11}+2 \hat{\beta}_{1} \hat{\beta}_{2} v_{12}+\hat{\beta}_{1}^{2} v_{22}\right)$,

where the $v_{i j}$ are the elements of $\widehat{V(\hat{\beta})}$. These statistics are clearly not identical. : Moreover, infinitely many other parameterizations equivalent to (2) and (3) can .. be calculated, each with their own corresponding test statistic. 
A Monte Carlo experiment was used to compare the relative performance of $W^{A}$ and $W^{B}$ in small samples. ${ }^{1}$ The data were generated artificially using (1) above with the null hypothesis true, with $x_{1}$ and $x_{2}$ obtained using a first-order vector autoregressive process with an own-lag coefficient of 0.6 and a cross-coefficient of 0.3 . The random component of each generated $x$ and the $\varepsilon_{t}$ were each drawn (independent $1 y$ ) from $a$ random standard normal deviate generator. ${ }^{2}$ The number of rejections at the 5 percent level of confidence are reported in Table 1 . Note that when the null hypothesis is true, one would expect 50 rejections out of 1000 with a 95 percent confidence interval $[36,64]$.

The table indicates that there are sharp differences between the test results. In particular, under the null hypothesis as $\beta_{2}$ approaches zero, $W^{A}$ tends to perform poorly in small samples, so that when $\beta_{2}=.1$, $W^{A}$ rejects 293 times compared to 65 times by $w^{B}$. Given that $\beta_{2}$ appears in the denominat or of $g^{A}$, one might perhaps expect this. Nevertheless, $W^{A}$ is a valid Wald test despite the fact that $g^{A}$ has no derivative at $\beta_{2}=0$, because the asymptotic distribution of the test is obtained only under the null hypothesis (see pages $445-446$ of Wald, 1943.. or the heuristic derivation of Silvey, 1975, pages 115-116). In this case the null hypothesis specifically precludes $\beta_{2}=0$. But clearly as $\beta_{2}$ approaches zero, there is an approximate violation of the required continuity of derivatives (Wald, 1943, p. 463). This causes practical difficulties without changing the theoretical asymptotic justification of the test in any way. 3

In addition, the table shows that the performance of the test $s$ alsodiffer when the null hypothesis is false. Under one parameter setting, $W^{A}$ performs better than $W^{B}$ in small samples, in the other, $W^{A}$ performs very poorly. 
Two other important results are observed but not presented in the table. First, under the null hypothesis, as $\beta_{2}$ becomes larger than one, $W^{A}$ and $W^{B}$ still perform similarly. Second, there are numerous conflicts between the tests, even when their distributions are similar. For example with $\beta_{1}=\beta_{2}=1$ and $N=20, W^{A}$ and $W^{B}$ are in conflict five percent of the time; this drops to one half of a percent at $N=500$.

\section{Concluding Remarks}

The results above are selected from the much more detailed and extensive study in Gregory and Veall (1984)' where several different assumptions about coefficients, variances and data generating processes are considered. In addition, the experiments are extended to Wald-testing of: common factor restrictions (Hendry and Mizon, 1978), nonlinear restrictions of rational expectations models (Hoffman and Schmidt, 1981), and an economic example concerning the impact of money supply shocks. All of these results confirm the conclusion that there can be substantial differences and discrepancies in Wald tests of different but algebraically equivalent nonlinear restrictions. Overa11, the Monte Car lo evidence suggests that there is some statistical advantage in testing restrictions using mitiplicative forms. Formalizing this last result using Edgeworth expansions is a topic for future research. 
FOOTNOTES

${ }^{1}$ A referee suggests the following heuristic argument to explain intuitively why the tests are equivalent asymptotically but not in finite samples: as $g^{B}=\hat{\beta}_{2} g^{A}$, the tests would be identical if $G^{B}=\hat{\beta}_{2} G^{A}$. As $G^{B}=\left(\hat{\beta}_{2}, \hat{\beta}_{1}\right)$ and $G^{A}=\left(1,1 / \hat{\beta}_{2}^{2}\right)$, this requires $\hat{\beta}_{1}=1 / \hat{\beta}_{2}$, which holds asymptotically under the null hypothesis, but not in finite samples due to sampling error.

${ }^{2}$ Random normal deviates were generated using IMSL subroutine GGNML as implemented on the CDC Cyber 170/835 at the University of Western Ontario. Processes were started 20 periods before the first observations were set.

${ }^{3}$ As an example of a case where the Wald test can differ analytically from $W^{B}$ with no restrictions on the domain of $\beta_{1}$ or $\beta_{2}$, consider that $H_{0}: \beta_{1} \beta_{2}=1$ might be tested using $H_{0}^{C}: g^{C}\left(\beta_{1}, \beta_{2}\right)=e^{\beta_{1} \beta_{2}}-e=0$.

${ }^{4}$ In the Hoffman and Schmidt case of testing the rational expectations hypothesis, it was found that the great similarity in Monte Carlo results for the $W$ and LR tests (extended to the LM case in Gregory and Veal1, 1985) applies only to the multiplicative form. With the ratio form of the restriction they cite (p. 267) but do not use in their computer program, results in Gregory and Veall (1984) indicate no such similarity. We thank Professor Schmidt for providing us with the computer program used in their article. 


\section{REFERENCES}

Burguete, J. F., A. R. Gallant and G. Souza (1982), "On Unification of the Asymptotic Theory of Nonlinear Econometric Models," Econometric Review 1, 151-190.

Cox, D. R. and D. V. Hinkley (1974), Theoretical Statistics, Chapman and Ha11, London.

Gregory, A. W. and M. R. Veall (1984), "On Formulating Wald Tests of Nonlinear Restrictions," Working Paper No. 8401 , University of Western Ontario, January. and (1985), "A Lagrange Multiplier Test of the Restrictions for a Simple Rational Expectations Model," Canadian Journal of Economics, forthcoming.

Hendry, D. F. and G. E. Mizon (1978), "Serial Correlation as a Convenient Simplification, Not as a Nuisance: A Comment on a Study of the Demand for Money by the Bank of England," The Economic Journal 88, $549-563$

Hoffman, D. I. and P. Schmidt (1981), "Testing the Restrictions Implied by the Rational Expectations Hypothesis," Journal of Econometrics 15 , $265-287$

Silvey, S. D. (1975), Statistical Inference, Chapman and Hall, London. Wald, A. (1943), "Tests of Statistical Hypotheses Concerning Several Parameters When the Number of Observations is Large," Transactions of the American Mathematical Society 54, 426-482. 
TABLE 1

Number of Rejections at the 5 Percent Level in 1000 Trials Using Wald Tests of Simple Nonlinear Restrictions

\begin{tabular}{|c|c|c|}
\hline ase & $\begin{array}{c}\text { Form of } \\
\text { Restriction }\end{array}$ & $\mathrm{N}=20$ \\
\hline
\end{tabular}

Nul1 hypothesis true:

$\begin{array}{lrrrrrr}\beta_{1}=10.0, \beta_{2}=0.1 & \text { A } & 293 & 253 & 206 & 142 & 78 \\ \beta_{1}=5.0, \beta_{2}=0.2 & \text { B } & 65 & 62 & 63 & 64 & 39 \\ \beta_{1}=2.0, \beta_{2}=0.5 & \text { A } & 201 & 152 & 119 & 108 & 77 \\ \beta_{1}=1.0, \beta_{2}=1.0 & \text { B } & 64 & 58 & 57 & 48 & 56 \\ & \text { A } & 86 & 89 & 78 & 58 & 43 \\ & \text { B } & 61 & 53 & 71 & 64 & 40\end{array}$

Nul1 hypothesis false:

\begin{tabular}{|c|c|c|c|c|c|c|}
\hline$\beta_{1}=1.5, \beta_{2}=1.0$ & $\begin{array}{l}\text { A } \\
\text { B }\end{array}$ & $\begin{array}{l}443 \\
278\end{array}$ & $\begin{array}{l}554 \\
399\end{array}$ & $\begin{array}{l}833 \\
728\end{array}$ & $\begin{array}{l}985 \\
971\end{array}$ & $\begin{array}{l}1000 \\
1000\end{array}$ \\
\hline$\beta_{1}=1.0, \beta_{2}=0.5$ & $\begin{array}{l}\mathrm{A} \\
\mathrm{B}\end{array}$ & $\begin{array}{r}65 \\
584\end{array}$ & $\begin{array}{l}196 \\
775\end{array}$ & $\begin{array}{l}601 \\
943\end{array}$ & $\begin{array}{l}992 \\
998\end{array}$ & $\begin{array}{l}1000 \\
1000\end{array}$ \\
\hline
\end{tabular}

